\title{
Optimization of Galantamine Loaded Bovine Serum Albumin Nanoparticles by Quality by Design and Its Preliminary Characterizations
}

Aditi Poddar and Krutika K Sawant*

TIFAC Centre of Relevance and Excellence, Centre of PG Studies and Research, Faculty of Pharmacy, The Maharaja Sayajirao University of Baroda, Vadodara, Gujarat 390002, India

\begin{abstract}
Alzheimer's disease is a progressive neurodegenerative disorder having a severe socio-economical and medical burden all over the world. The objective of this work was to develop nanoparticulate drug delivery system for Galantamine Hydrobromide (GAL) with the aid of Quality by Design (QbD) for intranasal administration to improve its targeting to brain. Bovine Serum Albumin (BSA) nanoparticles were prepared by desolvation process. Quality Target Profile and Critical Quality Attribute was set, proper risk identification and risk analysis was conducted using the Failure Mode and Effect Analysis. After proper risk assessment, optimization of the formulation was done using the Box-Behnken Design. Differential scanning calorimetry (DSC) and X-ray diffraction (XRD) were conducted. The characterization was done by studying the entrapment efficiency, particle size, zeta potential, transmission electron microscopy and atomic force microscopy. Drug amount, $\mathrm{pH}$ of aqueous phase, addition rate of desolvating agent was identified as parameters for Box-Behnken Design. The DSC of GAL nanoparticles did not show any peak at the melting point of GAL, proving its entrapment. Entrapment efficiency upto $78 \%$ could be achieved. A negative zeta potential was observed for GAL nanoparticles, ranging from $-13 \mathrm{mV} \pm 0.25$ to $-11 \mathrm{mV} \pm 0.32$. Nanoparticles were spherical, uniform in size with less than $200 \mathrm{~nm}$. The surface was smooth with few undulations, may be due to GAL particles adsorbed on the surface during ionic interaction between GAL and BSA. Therefore, GAL nanoparticles were formulated employing a very simple, cost effective desolvation method.
\end{abstract}

Keywords: Failure mode analysis; Quality by design; Galantamine nanoparticles; Bovine serum albumin; Atomic force microscopy

\section{Introduction}

Alzheimer's disease is a progressive neurodegenerative disorder which includes a variety of sporadic and/or familial, characterized by the persistent loss of neuronal activity. Alzheimer's disease affects 24.3 million people worldwide and hence becomes one of the most severe socio-economical and medical burden all over the world [1]. The disease burden of Alzheimer's goes beyond the cost of medicines/ therapy. Around the world, the global market for Alzheimer's disease is slated to increase to $\$ 13.3$ billion by 2023 [2]. In India, the total societal cost of dementia was estimated to be Rs. 206.11 billion [3].

With the proposition of Quality by Design (QbD) by US Food and Drug Administration (USFDA), the pharmaceutical product development has undergone a change in many ways. The focus of this concept is that quality should be built into a product with an understanding of the product and process by which it is developed and manufactured along with a knowledge of the risks involved in manufacturing the product and how best to mitigate those risk U.S. and Services [4]. QbD includes everything right from setting a predefined target quality, physicochemical, physiological, pharmacological and clinical considerations to obtain desired products that are safe and effective. The Federal government has been encouraging in many ways to utilize $\mathrm{QbD}$ for commercial development of a pharmaceutical product so that the final outcome is with fewer flaws and having better functional qualities.

In $\mathrm{QbD}$, product and process performance characteristics should be scientifically designed to meet specific objectives, and not empirically derived from the performance of test batches. Therefore, good product quality should represent an acceptably low risk of failing to achieve the desired clinical attributes [5]. The International Conference on Harmonization (ICH) Q8 [6], Q9 [7] and Q10 [8] guidelines describe principles and tools for the implementation and continuous improvement of $\mathrm{QbD}$. Amongst the various aspects that $\mathrm{QbD}$, Design of Experiment (DoE) plays a vital role in defining the variable that might contribute towards achieving the Quality Target Product Profile (QTPP), with less number of experiments.

Galantamine (GAL) belongs to the class of acetylcholine esterase inhibitors, the most successful class of therapeutic agents to decrease the progression of Alzheimer's disease. It is approved by the USA FDA and the European Medicines Agency for the symptomatic treatment of Alzheimer's disease due to its ability to moderate acetylcholinesterase inhibition in the CNS. GAL is commercially available as tablets and oral suspension. However, when administered via the oral route, it leads to severe nausea and vomiting because of its motor and evacuative function on the intestinal tissues [9]. Additionally, recent reports have shown that Galantamine also has anti-amyloid activity [10]. But to fully exploit its potential, it has to be delivered properly and that necessitates surpassing the Blood Brain Barrier (BBB).

Biodegradable polymers are always preferred over nonbiodegradable ones for formulating nanoparticles. Bovine serum albumin (BSA) is a very versatile biodegradable polymer, for drug delivery because of its medical importance, abundance, low cost, ease

*Corresponding author: Krutika K Sawant, Faculty of Pharmacy, Kalabhavan, The Maharaja Sayajirao University of Baroda, Vadodara 390001, Gujarat, India, Tel: 2652434187; E-mail: dr_krutikasawant@rediffmail.com

Received: September 01, 2017; Accepted: September 13, 2017; Published: September 20, 2017

Citation: Poddar A, Sawant KK (2017) Optimization of Galantamine Loaded Bovine Serum Albumin Nanoparticles by Quality by Design and Its Preliminary Characterizations. J Nanomed Nanotechnol 8: 459. doi: 10.4172/2157. 7439.1000459

Copyright: $\odot 2017$ Poddar A, et al. This is an open-access article distributed under the terms of the Creative Commons Attribution License, which permits unrestricted use, distribution, and reproduction in any medium, provided the original author and source are credited. 
of purification, unusual ligand-binding properties and wide acceptance in the pharmaceutical industry [11].

Forneguera et al. [12] developed PLGA nanoparticles of GAL to be administered by intravenous route, which is inconvenient and costly. Hanafy et al. [13] formulated chitosan based Gal nanoparticles for intranasal administration. However as both drug and polymer were cationic, the entrapment efficiency was very low. Moreover, these researchers did not apply the concept of QbD during their formulation development.

Hence, the objective of this work was to develop nanoparticulate drug delivery system for GAL with the aid of QbD for intranasal administration to improve its targeting to brain thereby reducing side effects. Such an endeavour is also expected to improve future market potential.

\section{Materials}

Galantamine hydrobromide (GAL) was kindly gifted by Sun Pharma Advanced Research Centre, Vadodara, India; Bovine Serum Albumin (Fraction V, 98\% purity) was purchased from Sigma Aldrich, India. All other reagents were of analytical grade.

\section{Methods}

\section{Preparation of Galantamine nanoparticles}

The nanoparticles were prepared by desolvation process. BSA ( 50 $\mathrm{mg}$ ) was dissolved in $4 \mathrm{ml}$ of distilled water. The $\mathrm{pH}$ of this solution was adjusted in between 8-9 with $0.1 \mathrm{M} \mathrm{Na}_{2} \mathrm{CO}_{3}$. Weighed amount of GAL (5 mg) was incubated with BSA solution for different duration of time (from 0.5 to 4 hour). This aqueous phase was desolvated with acetone by adding it dropwise, through a $1 \mathrm{ml}$ syringe fitted with a 23 gauge needle under magnetic stirring provided with heating plate (Remi Instruments, India). This system was subsequently heated to a temperature of $75^{\circ} \mathrm{C}$ for evaporation of acetone and thermal gelation of BSA. The nanoparticles obtained were separated by centrifugation at $4^{\circ} \mathrm{C}$ for $30 \mathrm{~min}$ at $35,000 \mathrm{RPM}$ (Optima L-100 XP Ultracentrifuge, Beckman, UK). The formed nanoparticles were then subjected to further characterization [14].
Quality target profile (QTPP): The quality target product profile (QTPP) is "a prospective summary of the quality characteristics of a drug product that ideally will be achieved to ensure the desired quality, taking into account safety and efficacy of the drug product.”The QTPP is an indispensable part of a $\mathrm{QbD}$ approach and the design of the product is based on it [15].

Risk identification: Before the GAL nanoparticles were prepared; the critical qualities to be monitored were identified and termed as Critical Quality Attributes. These are the identification of attributes related to the formulation and the process parameters regarding the above mentioned manufacturing method. An Ishikawa diagram (Fishbone diagram) was constructed to have a better understanding of the CQAs and the potential risks. On the basis of prior scientific knowledge, average particle size and encapsulation efficiency were considered as important CQAs of GAL nanoparticles as these parameters are likely to contribute substantially to the therapeutic efficacy [16].

Failure mode and effect analysis (FMEA) method is another way of conducting risk analysis for a product to be developed. In this type of analysis, each variable (which has a potential to cause failure) was scored in terms of severity (S), detectability (D) and probability (P). From these the Risk Priority Number was calculated $[17,18]$.

The factors with high RPN were subjected to further studies to ensure product safety and efficacy while those with lower RPN were eliminated from further studies. The QTPP and CQA for Galantamine nanoparticles are shown in Table 1.

Risk analysis by Plackett-Burman design: Based on the outcomes of FMEA, further analysis of risk was studied using the Plackett-Burman Design $[19,20]$. A total of six factors were selected from FMEA studies. The factors and their levels are shown in Table 2. These factors were varied at two levels each using the Design Expert software (Version 7.1), wherein 12 experimental runs were generated and executed in a randomized manner. The experimental design matrix for running the experiments is shown in Table 3.

For statistical analysis of the design, ANOVA and coefficients of quadratic equation were considered for both the responses viz particle size and entrapment efficiency. The values for the levels were determined based on preliminary experiments.

\begin{tabular}{|c|c|c|}
\hline QTPP Elements & Target & Justification \\
\hline Target patient population & Adults & Alzheimer's disease affects adults irrespective of the cause. \\
\hline Route of administration & Nasal & Surpass the gastric irritation, help in brain targeting. \\
\hline Dosage form & $\begin{array}{l}\text { White to off white pharmaceutically elegant } \\
\text { lyophilized cake that can be easily reconstituted. }\end{array}$ & $\begin{array}{l}\text { Required for long term stability of nanoparticles and good } \\
\text { appearance of the product. }\end{array}$ \\
\hline Dosage design & Nasal spray & $\begin{array}{l}\text { Required for spraying the dispersed nanoparticles to the dorsal part } \\
\text { of nasal cavity. }\end{array}$ \\
\hline Pharmacokinetics & $\begin{array}{l}\text { Acceptable parameters to demonstrate } \\
\text { therapeutic effect. }\end{array}$ & Needed to understand the bioavailability after nasal administration. \\
\hline Stability & At least 1 year stability in lyophilized condition & $\begin{array}{l}\text { Needed for quality and efficacy throughout the shelf life of the } \\
\text { product }\end{array}$ \\
\hline Container Closure System & $\begin{array}{l}\text { Container closure system qualified as suitable } \\
\text { for this drug product }\end{array}$ & $\begin{array}{l}\text { Needed to achieve the target shelf-life and to ensure proper integrity } \\
\text { of the product. }\end{array}$ \\
\hline Alternative method of administration & None & It is a suggested QTPP by the US FDA \\
\hline \multicolumn{3}{|l|}{ CQA } \\
\hline Particle Size & Less than $200 \mathrm{~nm}$ & Needed for efficient nose to brain targeting. \\
\hline Zeta Potential & In the range of \pm 20 to $\pm 30 \mathrm{mv}$ & Provides stability to nanoparticles. \\
\hline Entrapment Efficiency & Above $50 \%$ & $\begin{array}{l}\text { Required for increased drug loading and hence reducing bulk of } \\
\text { formulation to be administered. Also, avoid drug wastage. }\end{array}$ \\
\hline Form of the drug in nanoparticles & Amorphous & Better dissolution and absorption. \\
\hline Cellular Toxicity & Non toxic & No negative effects on the cells at the site of nanoparticle uptake. \\
\hline
\end{tabular}

Table 1: Quality target product profile and critical quality attributes for Galantamine nanoparticles $[8,15,18]$. 
Citation: Poddar A, Sawant KK (2017) Optimization of Galantamine Loaded Bovine Serum Albumin Nanoparticles by Quality by Design and Its Preliminary Characterizations. J Nanomed Nanotechnol 8: 459. doi: 10.4172/2157-7439.1000459

Page 3 of 10

\begin{tabular}{|c|c|c|c|}
\hline \multirow[t]{2}{*}{ Factors } & \multirow[t]{2}{*}{ Units } & \multicolumn{2}{|c|}{ Levels } \\
\hline & & Low & High \\
\hline Drug amount $\left(\mathrm{X}_{1}\right)$ & $\mathrm{mg}$ & 2 & 5 \\
\hline Polymer amount $\left(\mathrm{X}_{2}\right)$ & $\mathrm{mg}$ & 20 & 50 \\
\hline $\mathrm{pH}$ of aqueous phase $\left(\mathrm{X}_{3}\right)$ & & 4.5 & 8 \\
\hline Incubation time $\left(\mathrm{X}_{4}\right)$ & $\mathrm{hr}$ & 3 & 24 \\
\hline Rate of addition of desolvating agent $\left(\mathrm{X}_{5}\right)$ & $\mathrm{ml} / \mathrm{min}$ & 0.25 & 0.5 \\
\hline Speed of stirring $\left(\mathrm{X}_{6}\right)$ & RPM & 300 & 600 \\
\hline
\end{tabular}

Table 2: Factors and their respective levels for Plackett Burman design.

\begin{tabular}{|c|c|c|c|c|c|c|}
\hline \multirow[t]{2}{*}{ Batch Code } & \multicolumn{6}{|c|}{ Factors } \\
\hline & $\begin{array}{l}\text { Drug amount (mg) } \\
\qquad\left(X_{1}\right)\end{array}$ & $\begin{array}{l}\text { Polymer amount (mg) } \\
\left(\mathrm{X}_{2}\right)\end{array}$ & $\begin{array}{l}\text { pH of aqueous phase } \\
\left(\mathrm{X}_{3}\right)\end{array}$ & $\begin{array}{l}\text { Incubation time } \\
\text { between drug and } \\
\text { polymer }(\mathrm{hr})\left(\mathrm{X}_{4}\right)\end{array}$ & $\begin{array}{l}\text { Rate of addition of } \\
\text { desolvating agent } \\
(\mathrm{ml} / \mathrm{min})\left(\mathrm{X}_{5}\right)\end{array}$ & $\begin{array}{l}\text { Speed of stirring } \\
\text { (RPM) }\left(X_{6}\right)\end{array}$ \\
\hline PB1 & 2 & 50 & 8 & 24 & 0.25 & 600 \\
\hline PB2 & 2 & 50 & 4.5 & 24 & 0.5 & 600 \\
\hline PB3 & 2 & 20 & 4.5 & 3 & 0.25 & 600 \\
\hline PB4 & 5 & 50 & 4.5 & 3 & 0.25 & 300 \\
\hline PB5 & 2 & 20 & 8 & 3 & 0.5 & 300 \\
\hline PB6 & 5 & 20 & 8 & 24 & 0.5 & 600 \\
\hline PB7 & 5 & 50 & 4.5 & 24 & 0.5 & 300 \\
\hline PB8 & 2 & 20 & 4.5 & 24 & 0.25 & 300 \\
\hline PB9 & 5 & 20 & 4.5 & 3 & 0.5 & 600 \\
\hline PB10 & 2 & 50 & 8 & 3 & 0.5 & 300 \\
\hline PB11 & 5 & 20 & 8 & 24 & 0.25 & 300 \\
\hline PB12 & 5 & 50 & 8 & 3 & 0.25 & 600 \\
\hline
\end{tabular}

Table 3: Design Matrix for Plackett-Burman Design.

\section{Parameters}

Drug amount (mg)

$\mathrm{pH}$ of aqueous phase

Rate of addition of desolvating agent $(\mathrm{ml} / \mathrm{min})$

Table 4: Fixed process and formulation parameters for Box-Behnken design.

Optimization of GAL nanoparticles using Box-Behnken design: After assessing the critical formulation and process variables using Plackett-Burman Design, further optimization of the formulation was done by selecting the Box-Behnken Design (Design Expert, Ver 7.1). Here, a three-factor three-level design matrix was applied for optimization of GAL nanoparticles, leading to 17 experiments. The levels were assigned the same values as in Plackett-Burman Design. Also, the experiment runs were taken in a randomized manner to minimize associated errors.

Three variables out of the six variables studied in Plackett-Burman Design were found to be statistically insignificant towards the response variables; particle size and entrapment efficiency. Therefore their values were fixed and not included in the optimization by Box-Behnken Design (Table 4).

The factors and their three levels are shown in Table 5 and the design matrix for Box-Behnken Design can be viewed in Table 6.

The quadratic equations were generated for each response viz; particle size $\left(\mathrm{Y}_{1}\right)$ and entrapment efficiency $\left(\mathrm{Y}_{2}\right)$ leading to the critical parameters. Additionally, ANOVA was applied to understand the significance of the variables and the model as a whole. All these evaluations, along with construction of contour plots and response surface graphs, led to the optimization of the formulation with respect to particle size $\left(\mathrm{Y}_{1}\right)$ and entrapment efficiency $\left(\mathrm{Y}_{2}\right)$.

The optimized GAL nanoparticle formulation was prepared and tested to evaluate the correlation between the predicted and the actual

\begin{tabular}{|l|c|c|c|}
\hline \multirow{2}{*}{ Factors } & \multicolumn{3}{|c|}{ Factors } \\
\cline { 2 - 4 } & High & Medium & Low \\
\hline Speed of stirring (RPM) [A] & 600 & 450 & 300 \\
\hline Polymer amount (mg) [B] & 20 & 35 & 50 \\
\hline Incubation time (hr) [C] & 3 & 4 & 6 \\
\hline
\end{tabular}

Table 5: The factors and their levels for Box- Behnken design.

\begin{tabular}{|c|c|c|c|}
\hline Batch code & $\begin{array}{c}\text { Speed of stirring } \\
\text { (RPM) [A] }\end{array}$ & $\begin{array}{c}\text { Polymer amount } \\
\text { (mg) [B] }\end{array}$ & $\begin{array}{c}\text { Incubation time } \\
\text { (hr) [C] }\end{array}$ \\
\hline BB 1 & 450 & 50 & 3 \\
\hline BB2 & 450 & 20 & 6 \\
\hline BB3 & 450 & 35 & 4.5 \\
\hline BB4 & 600 & 35 & 6 \\
\hline BB5 & 450 & 35 & 4.5 \\
\hline BB6 & 450 & 35 & 4.5 \\
\hline BB7 & 300 & 35 & 6 \\
\hline BB8 & 300 & 20 & 4.5 \\
\hline BB9 & 600 & 20 & 4.5 \\
\hline BB10 & 600 & 35 & 3 \\
\hline BB11 & 450 & 20 & 3 \\
\hline BB 12 & 450 & 35 & 4.5 \\
\hline BB13 & 450 & 50 & 6 \\
\hline BB14 & 600 & 50 & 4.5 \\
\hline BB15 & 450 & 35 & 4.5 \\
\hline BB16 & 300 & 35 & 3 \\
\hline BB17 & 300 & 50 & 4.5 \\
\hline
\end{tabular}

Table 6: Experimental matrix for Box-Behnken design.

values of the responses. The optimum formulation was further studied for its physicochemical properties.

\section{Characterization of GAL loaded nanoparticles}

Differential scanning calorimetry (DSC): DSC helps in identifying possible interaction between drug and BSA and the physical changes 
of the active and excipient during the process of formulation. DSC analyses of GAL, BSA, their physical mixtures and the nanoparticulate formulation were performed using a DSC-60 instrument (Shimadzu, Japan). Indium was used for internal calibration. The samples were loaded separately into aluminum pans and hermetically sealed. An empty pan was used as reference. The samples were scanned from $30^{\circ} \mathrm{C}$ to $240^{\circ} \mathrm{C}$ at a scanning rate of $20^{\circ} \mathrm{C} / \mathrm{min}$. Nitrogen gas was used for purging the sample holders at a flow rate of $50 \mathrm{~mL} / \mathrm{min}$.

$\mathrm{X}$-ray diffractometry (XRD): XRD was performed to understand the physical state of BSA and GAL before and after their incorporation into a formulation. Additionally, it provided information on physical characteristics of GAL after its interaction with BSA during formulation of nanoparticles. Powder X-ray diffraction (PXRD) pattern were recorded using $X$ ray diffractometer (Panalytical'sXpert Pro, Netherlands) using $\mathrm{Cu} \mathrm{k} 2 \alpha$ rays with a voltage of $45 \mathrm{kV}$ and a current of $40 \mathrm{~mA}$ in a flat plate $\theta / 2 \theta$ geometry, over $2 \theta$ ranges between $5-70^{\circ}$ and signals were collected for $20 \mathrm{~min}$. A sample equivalent to 60 mg was placed in the sample holder groove and tightly packed.

Entrapment efficiency: For entrapment efficiency, the dispersion of GAL loaded nanoparticles was centrifuged at $4^{\circ} \mathrm{C}$ for $30 \mathrm{~min}$ at 35,000 RPM (Optima L-100 XP Ultracentrifuge, Beckman, UK). The supernatant was separated from the formed pellet and analyzed for free GAL spectrophotometrically (UV 1800, Shimadzu, Japan) at $289 \mathrm{~nm}$ against standard calibration curve. The drug entrapment was calculated from the equation given below:

$$
\text { Entrapment Efficiency }=\frac{\text { Original amount of drug added }- \text { Amount of free drug }}{\text { Original amount of drug added }} \times 100
$$

Particle size: The size and polydispersity index of the nanoparticles were determined using Malvern Zetasizer NanoSeries nano-ZS (Malvern Instruments Limited, Worcestershire, UK). Each sample was diluted ten times with filtered distilled water to avoid multi scattering phenomena and placed in a disposable sizing cuvette. Polydispersity index was studied to determine the narrowness of the particle size distribution. The size analysis of a sample consisted of 3 measurements, and the results are expressed as mean size \pm SD.

Zeta potential: Zeta potential was also measured using a Zetasizer (NanoSeries nano-ZS Malvern Instruments Limited, Worcestershire, UK). Each sample was suitably diluted with filtered distilled water and placed in a disposable zeta cell. Zeta limits ranged from -200 to $+200 \mathrm{mV}$. The electrophoretic mobility $(\mu \mathrm{m} / \mathrm{sec})$ was converted to zeta potential by in-built software using Helmholtz-Smoluchowski equation. Average of 3 measurements of each sample was used to derive zeta potential

Transmission electron microscopy: The morphology, structure and size ofGAL nanoparticles were observed through Transmission Electron Microscope (TEM) (Tecnai 20, Phillips, Holland) operating at an accelerating voltage of $200 \mathrm{kV}$, providing magnification upto $7,50,000 x$. The suspension of nanoparticles was deposited on a 200 mesh form coated copper grid and scanned at various magnifications.

\section{Atomic force microscopy}

To understand the topography of the GAL loaded nanoparticles Atomic Force Microscope (AFM) (Bruker Dimension Icon, USA) equipped with ScanAsyst Software was used [21]. Sample was loaded on a Mica plate (MUSCOVITE MICA, V-1 quality, USA) and spread uniformly on its surface. The mica plate was stored in a desiccator without desiccant for $15 \mathrm{~min}$ to immobilize them onto the surface. This plate was then placed under the microscope, which was placed on a vibration free table with an insulation cover. After setting the desired field of vision, the silicon tip cantilever was allowed to oscillate in the tapping mode with a spring constant of approximately $0.05 \mathrm{~N} / \mathrm{m}$ and a resonance frequency of approximately $37 \mathrm{kHz}$. The AFM topography image which reflects the topographic features of the surface was obtained from the amplitude change of the cantilever oscillation.

\section{Results and Discussions}

\section{Formulation of GAL nanoparticles}

There are many reports, [22] on BSA nanoparticles for delivering anti-cancer drug. However, its potential use as a carrier for antiAlzheimer drug has been less tread upon. As mentioned earlier, the aim was to prepare nanoparticles with minimum steps and less number of excipients so that it's commercially feasible.

The method chosen was desolvation followed by thermal gelation. This method is simple and produces small nanoparticles with a narrow distribution and very low polydispersity index Qi, Guo [23]. It is a well proven fact that the mean diameter of nanoparticles administered intranasally should be small to encourage endocytic pathways. In general, nanoparticles of adiameter $<200 \mathrm{~nm}$ are efficiently internalized by clathrin-mediated endocytosis [13]. Therefore, it's very crucial to control formulation and process parameters for obtaining desired particle size.

Preliminary studies for formulation of GAL nanoparticles: The choice of BSA as a polymer for formulation of GAL nanoparticles, served two purposes, first it being an anionic polymer at alkaline $\mathrm{pH}$, the chances of entrapment of cationic Galantamine increases. Secondly, BSA itself has a stabilizing effect in an aqueous media, therefore there is no requirement of an additional excipient like surfactant to stabilize the system [24]. Hence, the formulation remains simple, requires minimum excipents and doesn't involve complex steps.

Through preliminary studies for formulation, polymer amount, drug amount, rate of addition of desolvating agent, $\mathrm{pH}$ of the aqueous phase, incubation time between drug and polymer were some of the factors that were found to influence the formation of GAL nanoparticles.

Risk Identification and risk analysis: Ishikawa diagram (Fish bone analysis) shown in Figure 1A and 1B, aides in identification of the CQAs of a formulation and also helps in visualizing all the possible risks involved to achieve the CQAs. Here, the CQAs were particles size and entrapment efficiency. Both of these are of great significance when they are correlated with the administration route

For intranasal administration to brain, particle size plays a pivotal role in carrying the drug to its target by transcellularly transporting through olfactory neurones to the brain. Therefore it was aimed to obtain nanoparticles with size less than $200 \mathrm{~nm}$.

On the other hand, high encapsulation efficiency can help in reducing bulk of formulation to be administered and avoiding drug wastage during formulation. Hence it was identified as a CQA to be optimized for ensuring high entrapment efficiency.

Additionally, the technique of Failure Mode Effect Analysis was applied to understand the possible failures in the design of the formulation. This was done by means of ranking the failures with the help of RPN.

The RPN was calculated by scoring based on Severity, Detectability and Probability. These parameters were ranked, wherein 4 was the worst case, 1 was the best and 2 as moderate value. From these parameters, 
Citation: Poddar A, Sawant KK (2017) Optimization of Galantamine Loaded Bovine Serum Albumin Nanoparticles by Quality by Design and Its Preliminary Characterizations. J Nanomed Nanotechnol 8: 459. doi: 10.4172/2157-7439.1000459
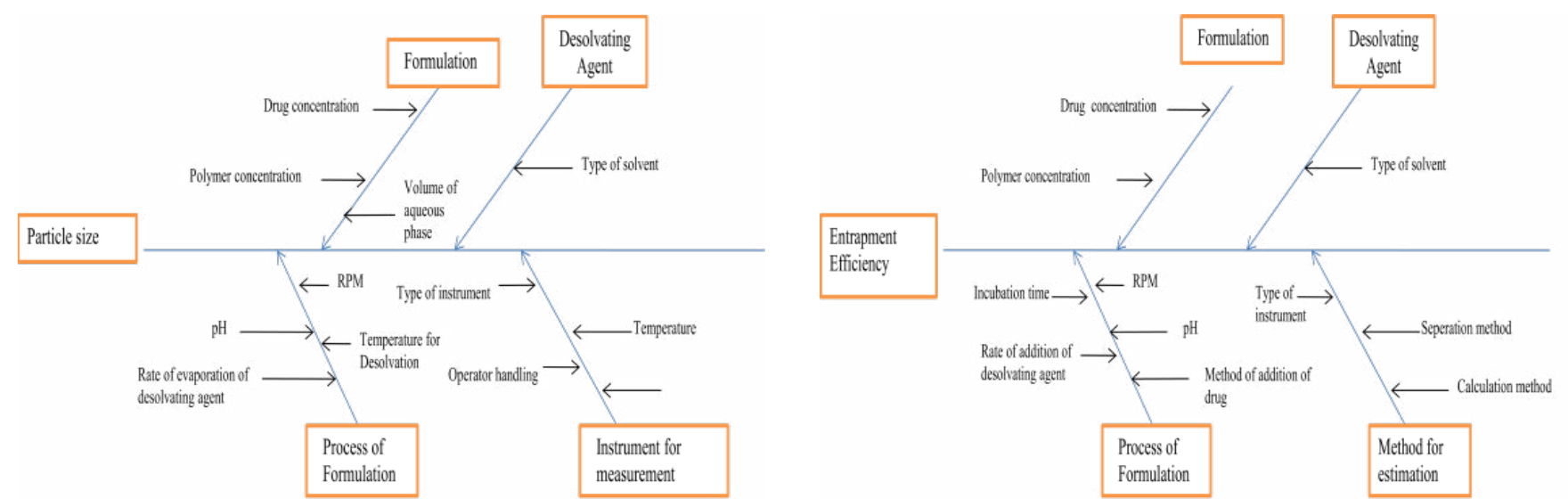

Figure 1: (A) Ishikawa diagram to understand the Critical Quality Attributes contributing to particle size. (B) Ishikawa diagram to understand the Critical Quality Attributes contributing to entrapment efficiency.
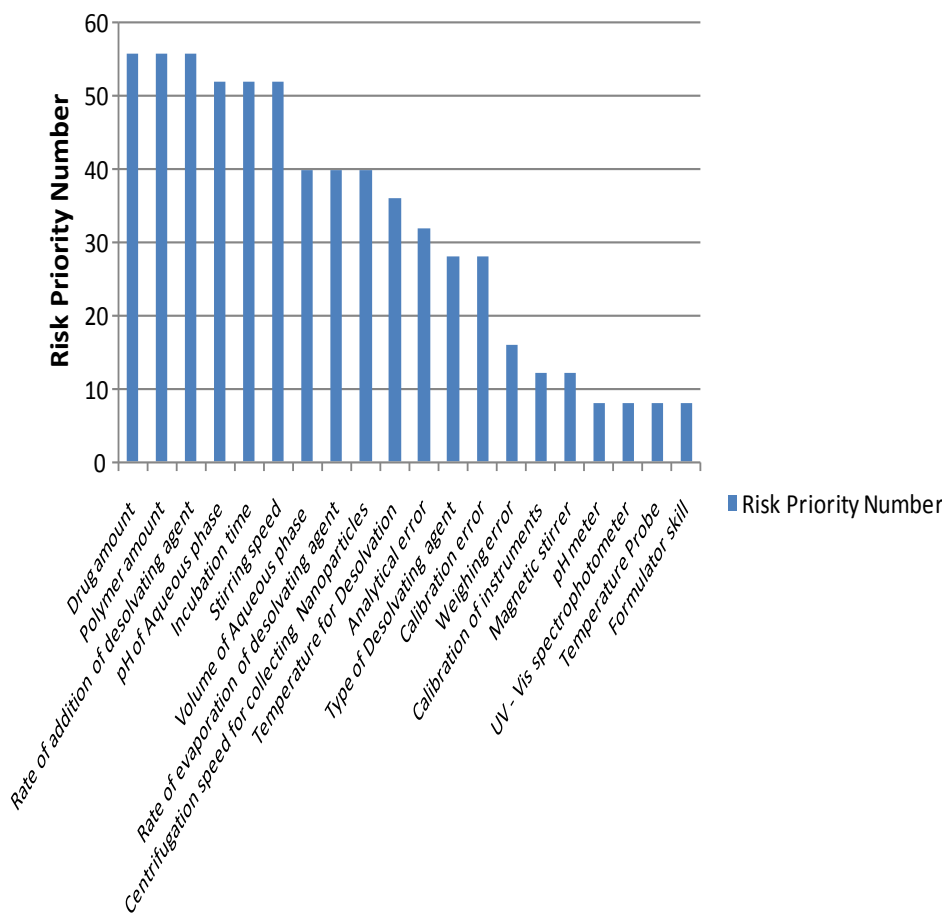

Attributes

Figure 2: Risk Priority Number associated with various attributes contributing to formulation of GAL nanoparticles.

the Risk Priority Number (RPN) was calculated using the formula:

$$
\mathrm{RPN}=\mathrm{S} \times \mathrm{D} \times \mathrm{P}
$$

Therefore the maximum value for RPN could be 64 and the minimum value could be 1 .

The threshold for RPN was fixed at 48 , and any formulation variable or process parameter with an RPN 48 or above was regarded as a potential critical factor [17]. Higher the RPN, higher is the risk and hence should be taken into account while designing a formulation.

The chart demonstrating all the possible risks leading involved in formulation of GAL nanoparticles is shown in Figure 2.
From Figure 2 it could be seen thatdrug and polymer amount, rate of addition of desolvating agent, $\mathrm{pH}$ of aqueous agent, incubation time between drug and polymer and stirring speed had the RPN higher than 48. Hence these factors were considered further for risk assessment and optimization.

Risk analysis by Plackett-Burman design: Based on the FMEA, statistical analysis of six factors was done by Plackett-Burman design. The outcome of the design for Particle size $\left(\mathrm{Y}_{1}\right)$ and Entrapment efficiency $\left(\mathrm{Y}_{2}\right)$ are shown in Table 7.

For particle size $\left(\mathrm{Y}_{1}\right)$, the percent contribution of speed of stirring, incubation time between the drug and polymer, drug amount and 


\begin{tabular}{|c|c|c|}
\hline Batch code & Particle size in $\mathbf{n m}\left(\mathbf{Y}_{\mathbf{1}}\right)^{*}$ & Entrapment efficiency in $\%\left(\mathbf{Y}_{2}\right)^{*}$ \\
\hline PB1 & $385.14 \pm 7.12$ & $40.12 \pm 3.93$ \\
\hline PB2 & $450.12 \pm 8.14$ & $0.16 \pm 0.02$ \\
\hline PB3 & $234.74 \pm 5.36$ & $2.57 \pm 0.17$ \\
\hline PB4 & $512.26 \pm 8.54$ & $6.4 \pm 0.09$ \\
\hline PB5 & $670.89 \pm 5.31$ & $64.12 \pm 2.83$ \\
\hline PB6 & $280.73 \pm 7.06$ & $24.17 \pm 0.32$ \\
\hline PB7 & $575.23 \pm 6.97$ & $1.39 \pm 0.04$ \\
\hline PB8 & $731.49 \pm 4.38$ & $5.17 \pm 0.23$ \\
\hline PB9 & $300.14 \pm 9.13$ & $3.47 \pm 0.13$ \\
\hline PB10 & $743.54 \pm 11.78$ & $57.96 \pm 4.36$ \\
\hline PB11 & $845.61 \pm 10.46$ & $32.78 \pm 1.96$ \\
\hline PB12 & $110.22 \pm 9.18$ & $78.56 \pm 4.67$ \\
\hline
\end{tabular}

Table 7: Results of responses after Plackett-Burman design.

\begin{tabular}{|l|c|c|c|c|}
\hline \multirow{2}{*}{ Factors } & \multicolumn{3}{|c|}{ Particle size in $\mathbf{n m}\left(\mathbf{Y}_{\mathbf{1}}\right)$} & \multicolumn{2}{c|}{ Entrapment Efficiency $\left(\mathbf{Y}_{2}\right)$} \\
\cline { 2 - 5 } & \% Contribution & $\boldsymbol{p}$ Value & \% Contribution & $\boldsymbol{p}$ Value \\
\hline Drug amount $\left(\mathrm{X}_{1}\right)$ & 4.94 & 0.1771 & 0.53 & 0.5983 \\
\hline Polymer amount $\left(\mathrm{X}_{2}\right)$ & 1.16 & 0.4803 & 2.64 & 0.2589 \\
\hline $\begin{array}{l}\mathrm{pH} \text { of aqueous phase } \\
\left(\mathrm{X}_{3}\right)\end{array}$ & 0.76 & 0.5645 & 11.53 & 0.0404 \\
\hline Incubation time $\left(\mathrm{X}_{4}\right)$ & 6.83 & 0.1240 & 74.91 & 0.0006 \\
\hline $\begin{array}{l}\text { Rate of addition of } \\
\text { desolvating agent }\left(\mathrm{X}_{5}\right)\end{array}$ & 0.57 & 0.6167 & 0.20 & 0.0443 \\
\hline Speed of stirring $\left(\mathrm{X}_{6}\right)$ & 75.72 & 0.0017 & 0.34 & 0.812 \\
\hline
\end{tabular}

Table 8: Statistical analysis of effect of variables on responses for Plackett-Burman design.

polymer amount respectively was the maximum. The $\mathrm{p}$ value for the model was 0.0215 , while $\mathrm{F}$ value was 7.49 and hence significant. The $\mathrm{R}^{2}$ was 0.89 . Also, the model had fewer signals to noise ratio. The $p$ value for incubation time between the drug and polymer, drug amount and polymer amount was greater than 0.05 and hence was not significant in the designed model. Therefore, RPM of magnetic stirrer was singled out as the factor contributing to smaller particle size.

For entrapment efficiency $\left(\mathrm{Y}_{2}\right)$, the contribution of incubation time between drug and polymer, $\mathrm{pH}$ of aqueous phase and polymer amount respectively was highest. The $p$ value for model was 0.0062 , whereas $\mathrm{F}$ value was 10.57 which indicated the model was highly significant. The $\mathrm{pH}$ of the aqueous phase, incubation time between drug and polymer solution and rate of addition of desolvating agent were significant variables for entrapment efficiency. The $\mathrm{pH}$ of aqueous phase solution, when kept at an alkaline side lead to better entrapment since the isoelectric point of $\mathrm{BSA}$ is $\mathrm{pH} 4.5$. Hence, at alkaline $\mathrm{pH}$, anionic polymer reacted with the cationic drug to give high entrapment. Similarly, the time for which the drug and polymer was in contact prior to desolvation also plays an important role in improving the entrapment efficiency. The entrapment efficiency kept on increasing with an increase till 4-5 hours of incubation. Thereafter it reduced with the increase in incubation time.

After the risk assessment with Plackett-Burman design, the $\mathrm{pH}$ of the aqueous phase and rate of addition of desolvating agent was kept constant for optimization, since they were not as significant as incubation time for entrapment efficiency. Additionally, polymer amount was taken ahead for optimization, as this factor had a contribution in both the responses.

Optimization by Box-Behnken design: After determining the statistically significant and important formulation and process variables by Plackett-Burman design, a three-factor, three-level Box-Behnken design was applied to precisely understand the impacts of RPM of magnetic stirrer (A), polymer amount (B), and incubation time(C)on particle size and encapsulation efficiency of GAL nanoparticles. The results of experimental runs are given in Table 8.

From Table 9 it could be seen that the particle size of nanoparticles prepared with lower RPM was found to be more than $300 \mathrm{~nm}$, which was not desirable for intranasal administration. However, higher RPM resulted into nanoparticles with particles less than $200 \mathrm{~nm}$ the minimum being about $110 \mathrm{~nm}$.

During desolvation, higher RPM generated turbulence which prevented the formation of larger particles, if any as well as aggregation of particles [25]. This in turn helped to maintain the particle size within a narrow distribution range.

Also, if the incubation time is very high, there are chances that the ionic interaction between drug and polymer increase to such a level that might cause them to desolvate as agglomerates leading to large particle size. On the other hand, if the incubation time is very low, the ionic interaction might not be sufficient enough for proper particle formation.

The quadratic equation obtained for the design with respect to particle size was:

$\mathrm{Y}_{1}=362.56-188.89 \mathrm{~A}-47.18 \mathrm{~B}-45.62 \mathrm{C}-87.09 \mathrm{AB}-18.03 \mathrm{AC}+$ $44.63 \mathrm{BC}+80.92 \mathrm{~A}^{2}+21.71 \mathrm{~B}^{2}+23.87 \mathrm{C}^{2}$

Eqn. (3) shows that all three factors had a negative influence on size that is size decreased with increase in these variables. The most significant factor contributing to smaller particle size was the RPM of magnetic stirrer, followed by polymer amount and incubation time.

Moreover interaction terms $\mathrm{AB}$ and $\mathrm{AC}$ were also negative but a positive interaction was observed between $B$ (polymer amount) and $C$ (incubation time). In case of entrapment efficiency, polymer amount was the most critical variable (Table 9).

Greater polymer amount improves the probability of it surrounding the drug, as a consequent there are improved chances of drug entrapment. However, the polymer amount should be optimum so that the size of the nanoparticles is also under control.

\begin{tabular}{|c|c|c|}
\hline Batch Code & Particle Size in $\mathbf{n m}\left(\mathbf{Y}_{\mathbf{1}}\right)^{*}$ & Entrapment Efficiency in $\%\left(\mathbf{Y}_{\mathbf{2}}\right)^{*}$ \\
\hline BB 1 & $300.52 \pm 7.05$ & $20.14 \pm 0.63$ \\
\hline BB2 & $426.51 \pm 9.86$ & $15.23 \pm 0.89$ \\
\hline BB3 & $380.83 \pm 4.80$ & $39.45 \pm 2.35$ \\
\hline BB4 & $215.76 \pm 2.09$ & $22.19 \pm 1.54$ \\
\hline BB5 & $394.19 \pm 4.50$ & $41.23 \pm 1.51$ \\
\hline BB6 & $400.73 \pm 7.84$ & $43.57 \pm 2.29$ \\
\hline BB7 & $540.17 \pm 8.08$ & $19.27 \pm 0.38$ \\
\hline BB8 & $641.23 \pm 7.14$ & $28.78 \pm 1.34$ \\
\hline BB9 & $348.21 \pm 4.95$ & $25.14 \pm 1.89$ \\
\hline BB10 & $430.63 \pm 6.90$ & $18.59 \pm 0.95$ \\
\hline BB11 & $519.47 \pm 6.96$ & $14.29 \pm 0.37$ \\
\hline BB 12 & $379.36 \pm 2.50$ & $44.87 \pm 3.70$ \\
\hline BB13 & $386.1 \pm 2.32$ & $22.74 \pm 0.66$ \\
\hline BB14 & $114.98 \pm 1.16$ & $78.23 \pm 2.04$ \\
\hline BB15 & $257.69 \pm 2.32$ & $66.53 \pm 2.25$ \\
\hline BB16 & $682.91 \pm 7.89$ & $22.39 \pm 0.56$ \\
\hline BB17 & $756.38 \pm 6.04$ & $72.12 \pm 2.29$ \\
\hline${ }^{*}$ Experiments were performed in triplicate. & \\
\hline & & \\
\hline
\end{tabular}

Table 9: Results of responses for Box-Behnken design. 
Citation: Poddar A, Sawant KK (2017) Optimization of Galantamine Loaded Bovine Serum Albumin Nanoparticles by Quality by Design and Its Preliminary Characterizations. J Nanomed Nanotechnol 8: 459. doi: 10.4172/2157-7439.1000459

Page 7 of 10

The quadratic equation obtained for the design with respect to entrapment efficiency was:

$$
\mathrm{Y}_{2}=47.13+0.19 \mathrm{~A}+13.72 \mathrm{~B}+0.50 \mathrm{C}+2.44 \mathrm{AB}+1.68 \mathrm{AC}+0.415 \mathrm{BC}
$$$$
+3.22 \mathrm{~A}^{2}+0.71 \mathrm{~B}^{2}-29.74 \mathrm{C}^{2}
$$

Eqn. (4) indicates that though all three factors had positive influence on entrapment efficiency, polymer amount contributed most significantly to entrapment efficiency.

Moreover, positive interactions were seen between all three factors; meaning, higher the value of these coefficients, higher would be the entrapment.

The model was evaluated by coefficient of determination $\left(\mathrm{R}^{2}\right)$. The $\mathrm{R}^{2}$ values were 0.89 and 0.79 for particle size and entrapment efficiency respectively, indicating that the model fitted significantly and also that a large amount of variance in the response was explained by the model ( $89 \%$ for particle size and $79 \%$ for entrapment efficiency).

The ANOVA was applied for model tested for particle size and entrapment efficiency and the $p$ values were 0.0115 and 0.0485 indicating it's suitability to predict the effect of variables on the responses.

Table 10 shows that, the $p$ value for stirring speed is 0.003 , signifying its important role in reducing the particle size. Also, the $p$

\begin{tabular}{|l|c|c|c|c|}
\hline \multirow{2}{*}{ Factors } & \multicolumn{2}{|c|}{ Particle size in $\mathbf{n m}\left(\mathbf{Y}_{\mathbf{1}}\right)$} & \multicolumn{2}{c|}{ Entrapment Efficiency $\left(\mathbf{Y}_{\mathbf{2}}\right)$} \\
\cline { 2 - 5 } & Sum of Squares & $\boldsymbol{p}$ Value & Sum of squares & $\boldsymbol{p}$ Value \\
\hline Speed of Stirring (A) & 285431.7 & 0.0003 & 0.316012 & 0.9689 \\
\hline Polymer amount (B) & 17807.62 & 0.1507 & 1506.731 & 0.0268 \\
\hline Incubation time(C) & 16652.21 & 0.1627 & 2.02005 & 0.0214 \\
\hline AB & 30342.16 & 0.0732 & 23.76563 & 0.7362 \\
\hline AC & 1300.684 & 0.6760 & 11.2896 & 0.8160 \\
\hline BC & 7969.133 & 0.3163 & 0.6889 & 0.9541 \\
\hline$A^{2}$ & 27576.68 & 0.0847 & 43.75816 & 0.6487 \\
\hline$B^{2}$ & 1984.751 & 0.6069 & 2.145007 & 0.9191 \\
\hline$C^{2}$ & 2400.82 & 0.5722 & 3725.013 & 0.0032 \\
\hline
\end{tabular}

Table 10: Statistical analysis of effect of variables on responses for Box-Behnken design.

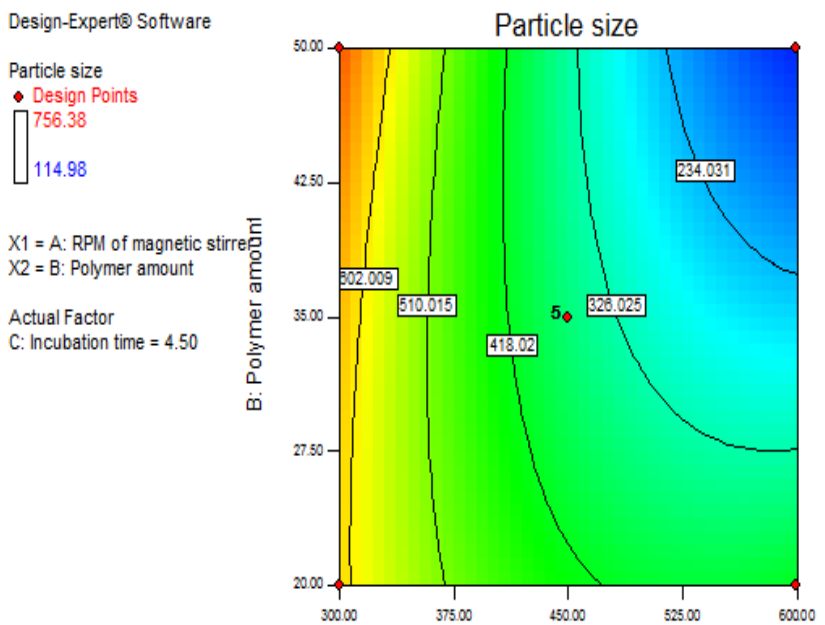

A: RPM of magnetic stirrer value for the incubation time is less than 0.05 , and hence is significant for entrapment efficiency. Proper incubation time allows proper ionic interaction between the drug and polymer. Higher the incubation, higher is the entrapment; however the incubation time had to be restricted to a certain limit so as to control the particle size.

To have a meticulous estimation of various factors on the responses, the significant variables affecting particle size and encapsulation efficiency were visually presented in contour and response surface plots (Figures 3 and 4 respectively).

Contour plots are a graphical representation of the relationship between three variables in two dimensions. These can be very helpful in illustrating the complete picture of the effect of two independent variables simultaneously (interactions) on the third variable (dependant variable) [26].

The contour plot for particle size can be seen in Figure 3, wherein the incubation time was set at the medium level of 4.5 hours and the other factors were varied. The incubation time was kept fixed since its value was least in eqn. (3). Polymer amount and speed of stirring were varied. Here, both the varying factors had an inverse relation with particle size.

The contour plot for entrapment efficiency is shown in Figure 4, the speed of stirring was set at a maximum value of 600 RPM. A direct relation was observed between both the factors; incubation time and polymer amount on the response that is entrapment efficiency.

The contour plots aided in providing an idea on what should be the values of varying parameters to achieve a particular particle size.

Similarly, considering other factors, the process can be optimized to achieve the desired product. Various contour plots can be generated between other variables studied in the design of experiments.

The design space for GAL nanoparticles was established targeting particle size lower than $140 \mathrm{~nm}$ and encapsulation efficiency higher than $75 \%$. For this, overlaid contour plots including both of the responses were constructed, which can be seen in Figure 5.

The overlaid contour plots were constructed by superimposing the

Design-Experte Software

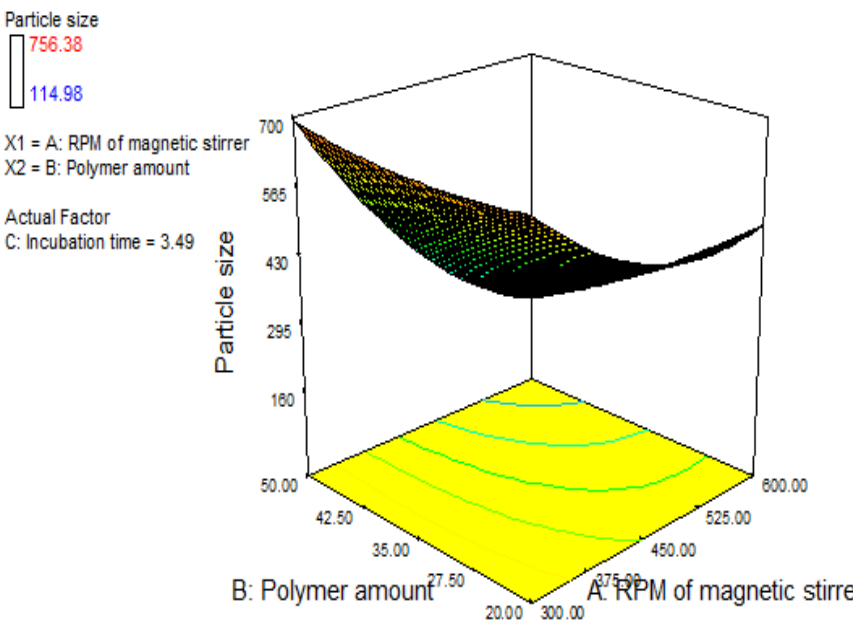

Figure 3: Response surface plot and contour plot for the significant variables on particle size. 
Citation: Poddar A, Sawant KK (2017) Optimization of Galantamine Loaded Bovine Serum Albumin Nanoparticles by Quality by Design and Its Preliminary Characterizations. J Nanomed Nanotechnol 8: 459. doi: 10.4172/2157-7439.1000459
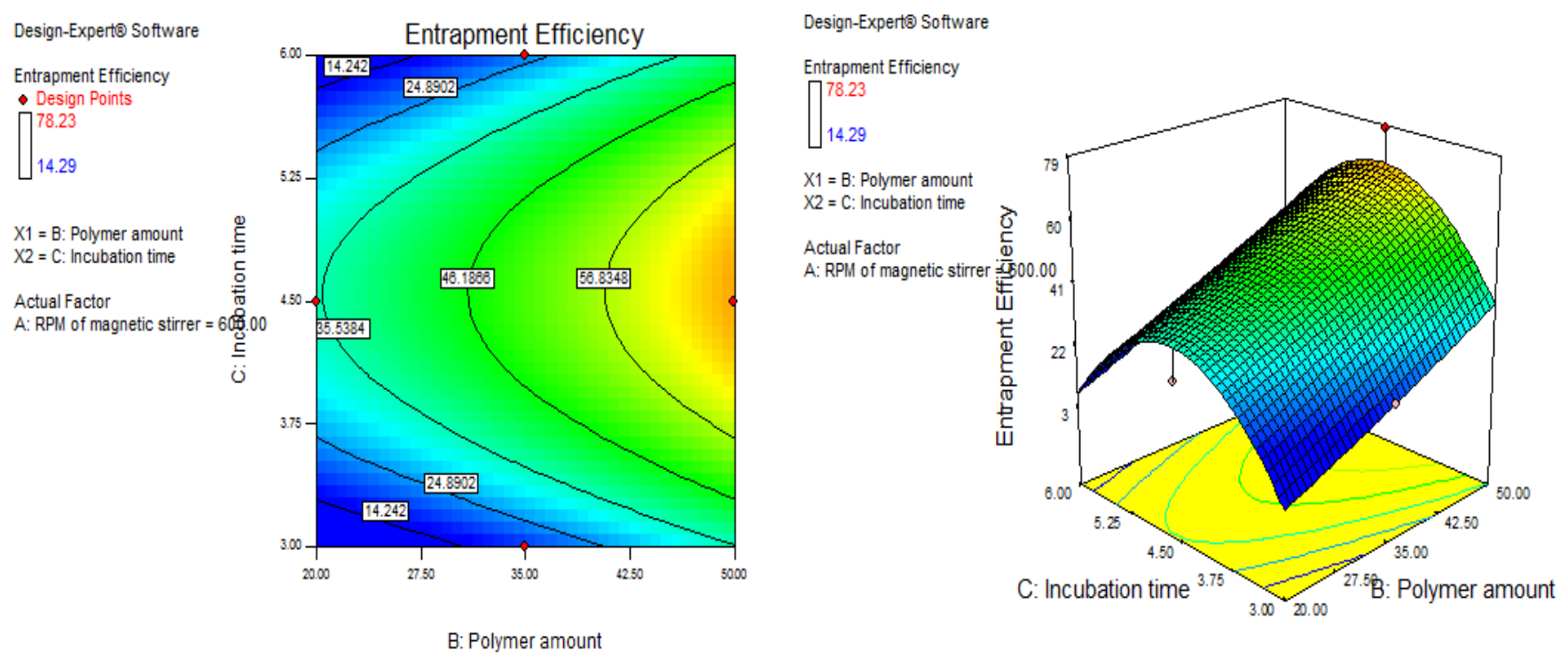

Figure 4: Response surface plot and contour plot for the significant variables on entrapment efficiency.

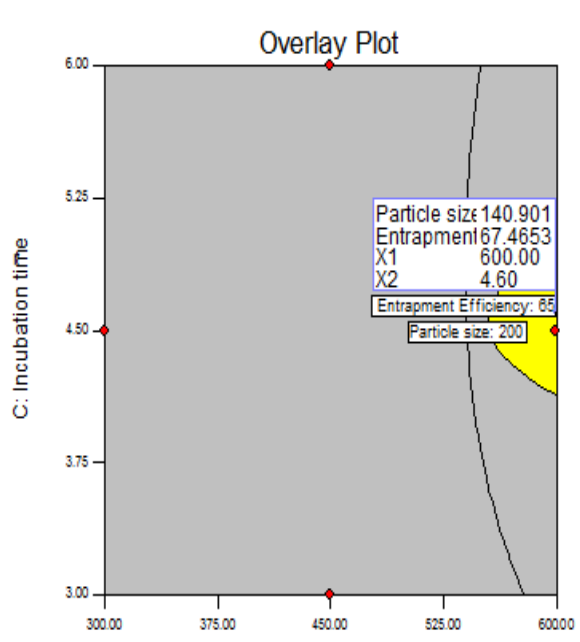

A: RPM of magnetic stirre

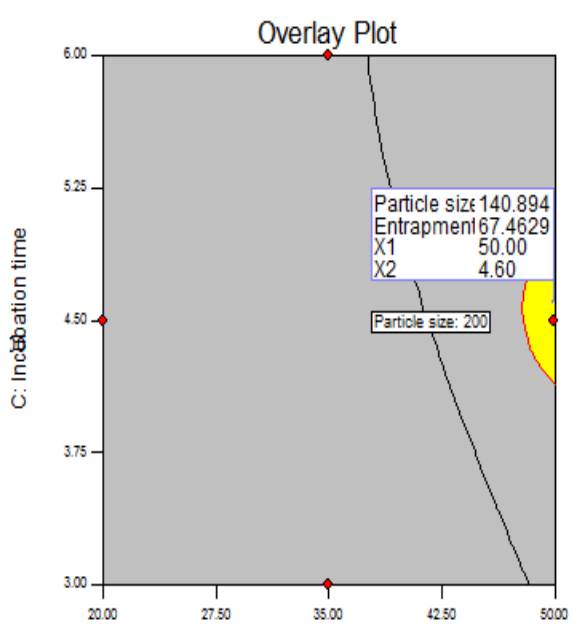

B: Polymer amount

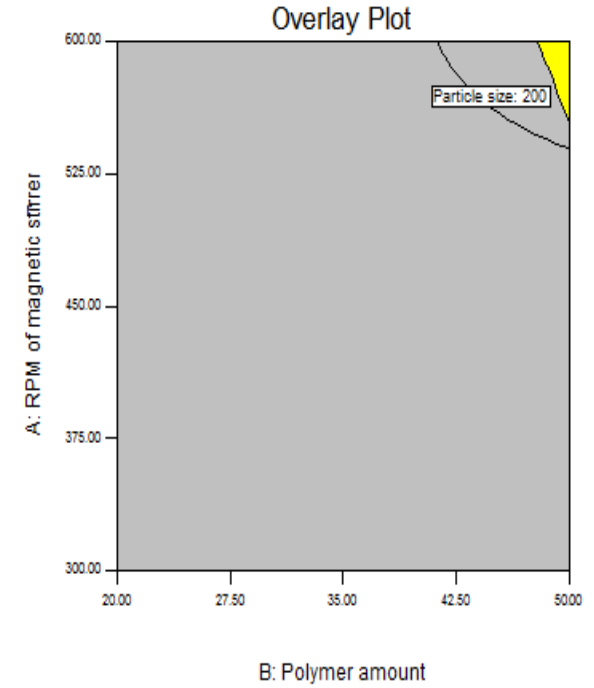

B: Polymer amount

Figure 5: Design space for GAL nanoparticles through overlay contour plots.

critical response contours of a contour plot. This resulted in the yellow region in Figure 5, describing an area of design space with feasible response values and grey region describing an area where response values did not fit the quality product criteria [26]. For instance, in Figure 5, to achieve a particle size of $140.92 \mathrm{~nm}$ and entrapment efficiency of $67.46 \%$ during formulation, the RPM of stirring should be 600 and the incubation time should be kept upto 4.6 hours.

\section{Characterization of GAL nanoparticles}

Differential scanning calorimetry (DSC): DSC provides details regarding the physical and energy state of a substance. Through DSC, it is possible to determine the physical state of drug substance in nanoparticle. The results of DSC are shown in Figure 6(i). Pure GAL 6 (i) (B) showed a sharp exothermic peak at $280^{\circ} \mathrm{C}$, representing its melting point as well as confirming its crystalline nature. The physical mixture of BSA and GAL showed characteristic peaks of both BSA and GAL 6(i) (C). The DSC of GAL nanoparticles 6 (i) (D) did not show any peak at the melting point of GAL demonstrating its encapsulation into polymer matrix and also the possibility of it being in amorphous state. While formulating a dosage form, it is preferred that the drug in the formulation is amorphous, resulting in better dissolution, absorption and bioavailability [23].

X-ray diffraction (XRD): As seen in Figure 6(ii). BSA showed a halo pattern confirming its amorphous nature. The XRD pattern of GAL showed sharp $2 \theta$ peak at $20.67^{\circ}$ with an area under the peak of 2200.83 confirming its crystallinity. The diffraction pattern of GAL nanoparticles showed diminished peaks of the drug with an area under peak of 147.54 which suggested the presence of crystal particles. However, since the value of area under peak for nanoparticles is lower than pure drug, the drug may exist in mixed form that is the crystalline 
Citation: Poddar A, Sawant KK (2017) Optimization of Galantamine Loaded Bovine Serum Albumin Nanoparticles by Quality by Design and Its Preliminary Characterizations. J Nanomed Nanotechnol 8: 459. doi: 10.4172/2157-7439.1000459

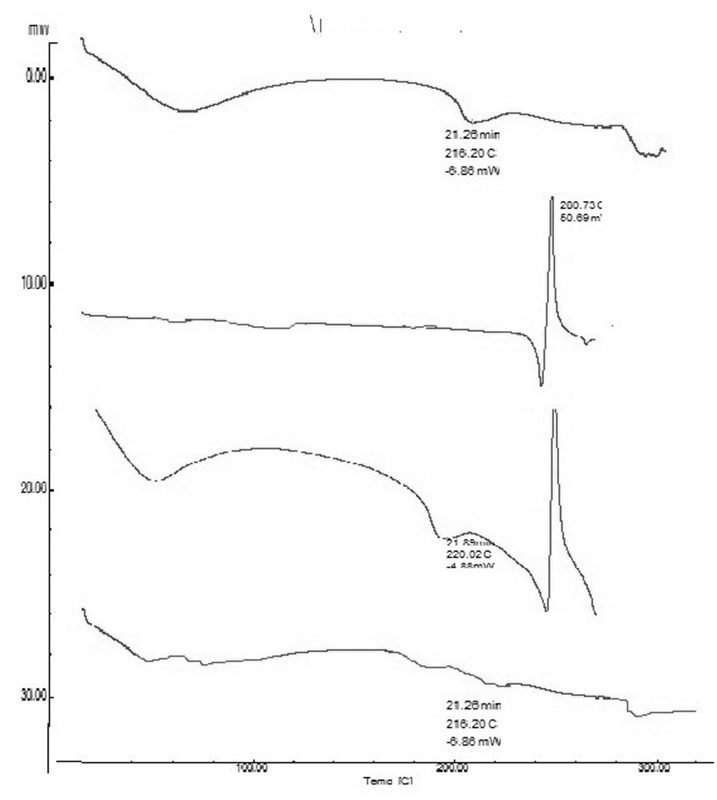

(i)

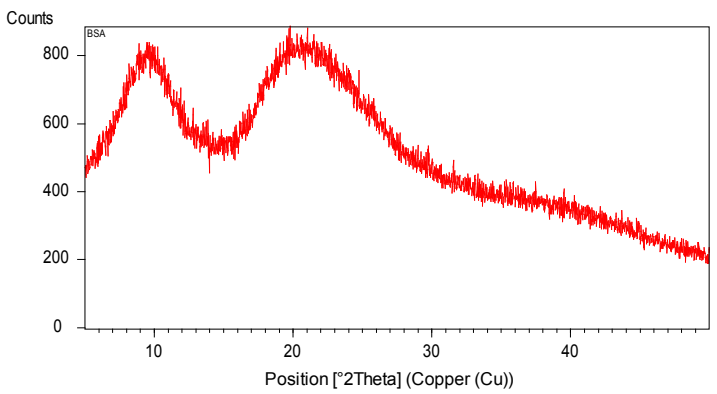

A

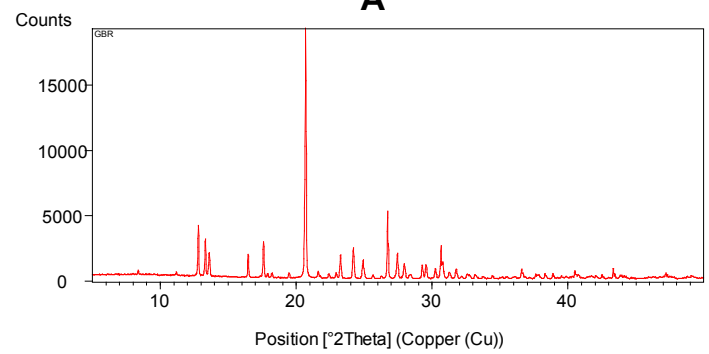

B

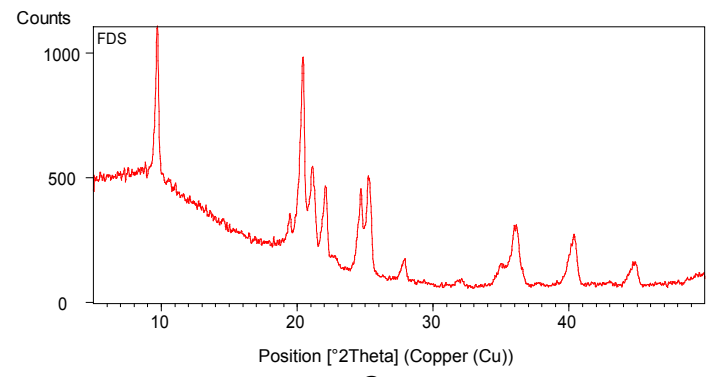

C

(ii)

Figure 6: (i) DSC of (A) BSA (B) Pure GAL (C) Physical mixture of GAL and BSA (D) GAL nanoparticles. (ii) XRD of (A) BSA (B) Pure GAL (C) GAL nanoparticles. nature is retained to some extent. Thermograms of DSC does not reflect this since crystallinities of under $2 \%$ cannot generally be detected by this method [26].

Zeta potential: Zeta potential is closely related to the stability of the nanoparticle system. The same type of surface charge could provide repulsion among the nanoparticles, maintaining an appropriate particle density and stability [23]. Here, a negative zeta potential was observed for GAL nanoparticles, ranging from $-13 \mathrm{mV} \pm 0.25$ to $-11 \mathrm{mV} \pm 0.32$.

The zeta potential of blank BSA nanoparticles ranged from -18 $\mathrm{mV} \pm 0.16$ to $-23 \mathrm{mV} \pm 0.30$. Thus, the GAL loaded nanoparticles had lower zeta potential than the blank nanoparticles indicating interaction between the negatively charged BSA and the positively charged drug, thereby reducing the zeta potential.

This low negative zeta potential along with small particle size was thought to be desirable prerequisites for good brain uptake [12].

Transmission electron microscopy: Figure 7 shows that the GAL nanoparticles were spherical, uniform in size and had size less than 200 $\mathrm{nm}$, indicating proper control over the process parameters like stirring speed.

Atomic force microscopy: Figure 8 shows the three dimensional AFM topography of GAL nanoparticles.

The surface was smooth with few undulations, which might suggest the presence of GAL particles adsorbed on the surface during the process of ionic interaction between GAL and BSA. This result is in line with the observations from zeta potential study wherein the GAL nanoparticles had lower zeta potential than blank BSA nanoparticles, suggesting some level of interaction.

\section{Conclusions}

GAL loaded BSA nanoparticles were formulated employing a very simple, cost effective desolvation method.

The method and the formulation variables like polymer amount, drug quantity, speed of stirring were critically assessed through QbD by proper risk identification, QTPP and CQAs prior to initiation of formulation. The optimization by Box-Behnken Design was done after statistical risk assessment by Plackett-Burman Design. Attributes like stirring speed, polymer amount, incubation time were found to contribute significantly towards smaller particle size and higher entrapment efficiency.

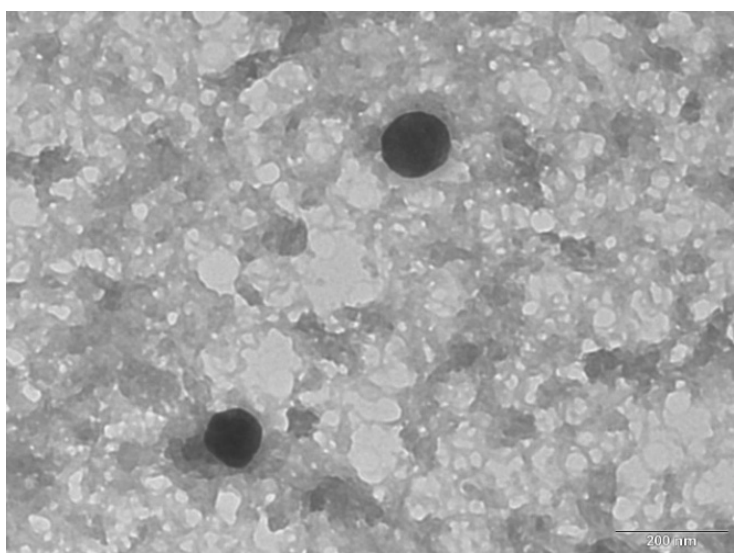

Figure 7: TEM of Formulation Code BB14. 
Citation: Poddar A, Sawant KK (2017) Optimization of Galantamine Loaded Bovine Serum Albumin Nanoparticles by Quality by Design and Its Preliminary Characterizations. J Nanomed Nanotechnol 8: 459. doi: 10.4172/2157-7439.1000459

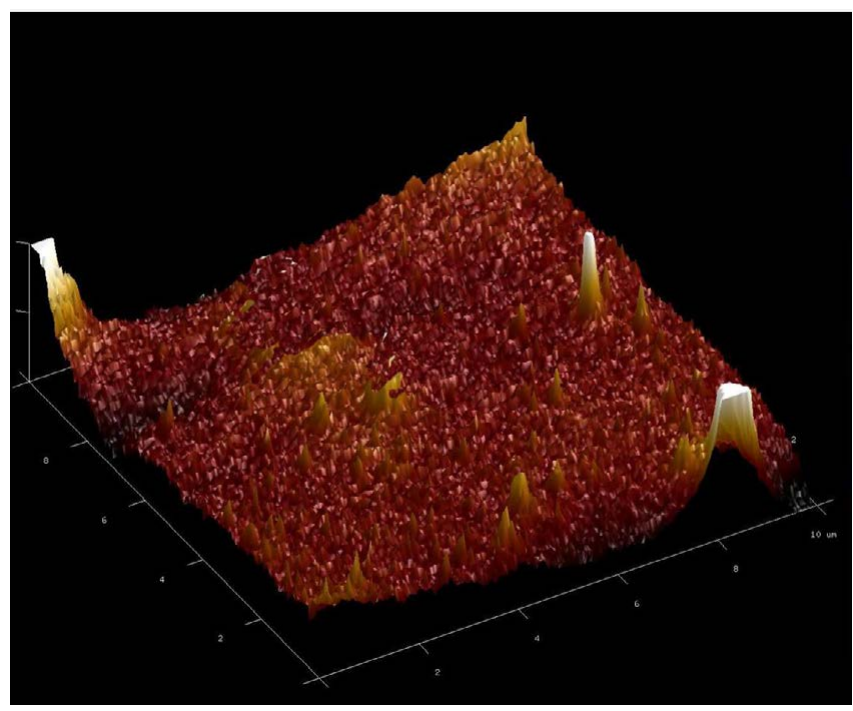

Figure 8: Detailed three dimensional AFM topography of GAL nanoparticles.

With the aid of prior knowledge and $\mathrm{QbD}$, a design space could be constructed for GAL nanoparticles with small particle size and high entrapment efficiency for better targeting and therapeutic effects.

The GAL nanoparticles were further characterized for zeta potential, shape and structural morphology by TEM and AFM. Studies through DSC and XRD, showed no incompatibility between the polymer and drug.

The drug release, ex vivo studies, cytotoxicity and in vivo studies are underway to further assess the GAL loaded BSA nanoparticles as a promising advanced drug delivery system against Alzheimer's disease.

\section{Disclosures}

The authors report no conflict of interest.

\section{Acknowledgements}

The authors are thankful to the University Grants Commission, India for funding the research, Sun Pharma Advanced Research Centre, India for providing a gift sample of GAL and CeNSE, Indian Institute of Science, India for AFM.

\section{References}

1. Ferri CP, Princ M, Brayne C, Brodaty H, Fratiglioni L, et al. (2005) Global prevalence of dementia: a Delphi consensus study. Lancet 366: 2112-2117.

2. Nicholsan KS (2015) Alzheimer's disease treatment market value will exceed $\$ 13$ billion. Bioscience Technology.

3. Sharma U (2011) Silent Killer in Express Pharma Pulse.

4. (2011) Guidance for Industry Process Validation: General Principles and Practices 22-27.

5. Jameel F, Khan M (2009) Quality-by-design as applied to the development and manufacturing of a lyophilized protein product. American Pharmaceutical Review 20-24.

6. Pharmaceutical Development (2009) International Conference on Harmonisation: Geneva, Switzerland.

7. Quality Risk Management (2005) International Conference on Harmonisation: Geneva, Switzerland.

8. Pharmaceutical Quality System (2008) International Conference on Harmonisation: Geneva, Switzerland.

9. De Caro V, Giandalia G, Siragua MG, Campsisi G, Giannola LI (2009) Galantamine delivery on Buccal mucosa: Permeation enhancement and design of matrix tablets. J Bioequiv Availab 1: 127-134.
10. Matharu B, Gibson G, Parsons R, Huckerby TN, Moore SA (2009) Galantamine inhibits beta-amyloid aggregation and cytotoxicity. J Neurol Sci 280: 49-58.

11. Elzoghby AO, Samy WM, Elgindy NA (2012) Albumin-based nanoparticles as potential controlled release drug delivery systems. J Control Release 157 168-182.

12. Fornaguera C, Feiner-Gracia N, Caldero G, Garcia-Celma MJ, Solans C (2015) Galantamine-loaded PLGA nanoparticles, from nano-emulsion templating, as novel advanced drug delivery systems to treat neurodegenerative diseases. Nanoscale 7: 12076-12084.

13. Hanafy AS, Farid RM, EIGamal SS (2015) Complexation as an approach to entrap cationic drugs into cationic nanoparticles administered intranasally for Alzheimer's disease management: preparation and detection in rat brain. Drug Dev Ind Pharm 41: 2055-2068.

14. Merodio M, Arnedo A, Renedo MJ, Irache JM (2001) Ganciclovir-loaded albumin nanoparticles: characterization and in vitro release properties. Eur $\mathrm{J}$ Pharm Sci 12: 251-259.

15. FDA (2012) Quality by Design for ANDAs: An Example for Immediate-Release Dosage Forms.

16. Vogt FG, Kord AS (2011) Development of quality-by-design analytical methods J Pharm Sci 100: 797-812.

17. Shuling K, Jing L, Jianping L, Junlin W, Zhao $Y$ (2014) A quality by design $(\mathrm{QbD})$ case study on enteric-coated pellets: Screening of critical variables and establishment of design space at laboratory scale. Asian Journal of Pharmaceutical Sciences 9: 268-278.

18. Pallagi E, Ambrus R, Szabo-Revesz P, Csoka I (2015) Adaptation of the quality by design concept in early pharmaceutical development of an intranasal nanosized formulation. Int J Pharm 491: 384-392.

19. Yerlikaya F, Ozgen A, Vural I, Guven O, Karaagaoglu E (2013) Development and evaluation of paclitaxel nanoparticles using a quality-by-design approach. J Pharm Sci 102: 3748-3761.

20. Rahman Z, Zidan AS, Habib MJ, Khan MA (2010) Understanding the quality of protein loaded PLGA nanoparticles variability by Plackett-Burman design. Int $J$ Pharm 389: 186-194.

21. Moribe K, Limwikrant W, Higashi K, Yamamoto K (2012) Structural evaluation of probucol nanoparticles in water by atomic force microscopy. Int $\mathrm{J}$ Pharm 427: $365-371$.

22. Dreis S, Rothweiler F, Michaelis M, Cinatl J Jr, Kreuter J (2007) Preparation, characterisation and maintenance of drug efficacy of doxorubicin-loaded human serum albumin (HSA) nanoparticles. Int J Pharm 341: 207-214.

23. Lisi Q, Yuanyuan G, Jingjing L, Dianrui L, Zhongxi Z (2014) Folate-modified bexarotene-loaded bovine serum albumin nanoparticles as a promising tumortargeting delivery system. Journal of Materials Chemistry B 2: 8361-8371.

24. Patel Y, Poddar A, Sawant K (2014) Improved Oral Bioavailability of Cefuroxime Axetil Utilizing Nanosuspensions Developed by Media Milling Technique. Pharmaceutical Nanotechnology 2: 75-86

25. Verma S, Lan Y, Gokhale R, Burgess DJ (2009) Quality by design approach to understand the process of nanosuspension preparation. Int J Pharm 377 185-198.

26. Grundlagen der Arzneiformenlehre (1999) Springer-Verlag Berlin Heidelberg. 